Gravitation $\&$ Cosmology, Vol. 00 (2008), No. 0 (00), pp. 0-00

(C) 2008 Russian Gravitational Society

\title{
PRIMORDIAL BLACK HOLE: MASS AND ANGULAR MOMENTUM EVOLUTION
}

\author{
G.S. Bisnovatyi-Kogan ${ }^{1 *} \dagger \ddagger$ and O.Yu. Tsupko ${ }^{2 *} \ddagger$ \\ '* Space Research Institute of Russian Academy of Science, Profsoyuznaya 84/32, Moscow 117997, Russia \\ $\dagger$ Joint Institute Nuclear Research, Dubna, Russia \\ $\ddagger$ Moscow Engineering Physics Institute, Moscow, Russia
}

\begin{abstract}
The evolution of the primordial low mass black hole (PBH) in hot universe is considered. Increase of mass and decrease of $\mathrm{PBH}$ spin due to the accretion of radiation dominated matter are estimated with using of results of numerical simulation of $\mathrm{PBH}$ formation and approximate relations for accretion to a rotating black hole. ${ }^{3}$
\end{abstract}

\section{Introduction}

'The possibility of formation of the primordial black holes $(\mathrm{PBH})$ with small masses at the early stages of Universe expansion was discussed in papers [1], [2], 'see also [3], [4], [5, 6]. Let us consider the early stages 'of expansion, and $\mathrm{PBH}$, massive enough for Hawking temperature $T_{H}=\frac{\hbar c^{3}}{8 \pi k G M}$ to be much less than environment temperature. In this case the main process of interaction between $\mathrm{PBH}$ and environment is accretion of the radiation dominated matter to $\mathrm{PBH}$. In approxima 'of steady flow, when the flow of mass $\dot{M} \equiv \frac{d M}{d t}$ is calculated with formulae for stationary flow of gas, at 'rest in infinity, to a gravitation center, the mass of $\mathrm{PBH}$ diverges if initial time of accretion is close to beginning of Universe expansion [1]. Authors [1] note that to answer the question whether accretion to $\mathrm{PBH}$ is 'catastrophically high the investigation of non-stationary problem is needed. Non-stationary problem is solved numerically in paper [7.

Mentioned above papers consider non-rotating black holes defined by Schwarzchild metric, and isotropic accretion of gas, which increases PBH mass and keeps angular momentum equal zero. But it is reasonable to assume formation of $\mathrm{PBH}$ with both mass and angular momentum. The presence of rotation is a proper feature of objects of different scales, beginning with elementary particles (spin) and up to rotation of macroobjects. As to $\mathrm{PBH}$, formation of $\mathrm{PBH}$ with both mass and angular momentum may result from simultaneous action of potential and vortex initial perturbations.

It is shown for the first time by Doroshkevich [8] that due to effects of the general theory of relativity accretion of particles (both non-relativistic and relativistic) to rotating objects leads in general case to decrease of angular momentum of the object. In papers [9], 10]

\footnotetext{
${ }^{1}$ e-mail: gkogan@iki.rssi.ru

e-mail: tsupko@iki.rssi.ru

3 Talk presented at the russian summer school-seminar "Modern theoretical problems of gravitation and cosmology" (GRACOS2007), September 9-16, 2007, Kazan-Yalchik, Russia
}

accretion of massive particles and photons to Kerr black hole is considered. In particular, in paper [9] author discusses the decrease of $\mathrm{PBH}$ angular momentum due to the isotropic accretion of photons.

In present paper increase of mass and decrease of $\mathrm{PBH}$ angular momentum are estimated taking into consideration non-stationarity of the problem (see also [11). We use results of numerical solution of non-stationary problem of PBH formation and dynamics [7] and approximate relations for accretion into a rotating black n hole 9 .

\section{Approximation of stationary accretion}

In approximation of stationary accretion one has the following equation for increase the PBH mass [1]-[4]:

$$
\frac{d M}{d t}=\frac{27}{4} \pi R_{G}^{2} c \rho_{r},
$$

where $M$ is black hole mass, $R_{G}=2 G M / c^{2}$ is its Schwarzchild radius, $\rho_{r}$ is the density of background matter. At the radiation dominated stages the density in Universe falls as $\rho_{r}=\frac{3}{32 \pi G t^{2}}$, what allows to integrate the equation (1). The integral depends weakly on upper bound in time, therefore one may put it equal to infinity. Then we have:

$$
M=\frac{M_{0}}{1-\frac{81}{32} \frac{G M_{0}}{c^{3} t_{0}}},
$$

where $M_{0}$ is the mass of black hole formed at the time $t_{0}$. Formula (2) diverges under $t_{0} \rightarrow \frac{81}{32} \frac{G M_{0}}{c^{3}}=\frac{81}{64} \frac{R_{G}}{c}$. Thus if at the time of black hole formation $t_{0}$ its gravitational radius $R_{G}$ was comparable with horizon radius $c t_{0}$, then in stationary approximation accretion would lead to very high increase of mass, as compared with initial. Note, that for $t \gg \frac{81}{32} \frac{G M_{0}}{c^{3}}$ the growth of mass of $\mathrm{PBH}$ is negligible, so for $M_{P B H} \sim 10^{15} \mathrm{~g}$ we can completely ignore it at $t \gg 10^{-20} \mathrm{~s}$ from the 
Big Bang. Because of decrease of PBH angular momentum due to accretion, it would lead us to conclusion about existence of almost non-rotating black holes, even if they had considerable specific angular momentum (non-dimensional) of the order of Kerr limit momentum $a_{\text {lim }}=\frac{J G}{M c^{3}}=1$ at the moment of formation. It is clear that the latter conclusion is associated with assumption of the stationary accretion and is not convincing. Relation between the mass of just formed PBH and the Universe horizon, which determines evolution of mass and angular momentum under the subsequent accretion, can be found only by solution of non-stationary problem.

\section{Estimations for increase of mass and decrease of angular momentum}

As shown in previous section, formula (2) doesn't lead to catastrophical accretion if the black hole mass is considerably smaller than $c^{3} t_{0} / G$. It means that black hole mass must be smaller than the mass contained within Universe horizon at the moment of time $t_{0}$. Non-stationary spherically symmetric problem of $\mathrm{PBH}$ formation as a result of evolution of initial strong perturbations relative to Friedman background model is solved numerically in paper [7]. It is found that at the time of its formation $\mathrm{PBH}$ will have mass considerably smaller than the mass within the cosmological horizon, therefore catastrophical accretion doesn't occur. This result is obtained for wide range of initial conditions. It also agrees with the result of qualitative analysis made in paper [2].

The main idea of the present paper is to estimate the angular momentum losses of PBH inherited at their birth, at the first stages of the Universe expansion. For that purposes we combine the results of the PBH mass evolution in hot Universe following from the numerical simulations (solution of non-stationary problem) and results for the angular momentum change due to accretion of the photons. We use the results of numerical calculations [7 to estimate the mass growth and PBH angular momentum loss, assuming the same rate of accretion. If the mass of formed PBH is $M_{0}=\eta \frac{32}{81} \frac{c^{3} t_{0}}{G}$, where $\eta$ is a non-dimensional coefficient, then in the absence of catastrophical accretion $(\eta \ll 1)$ we use the relation for stationary accretion (2) in order to estimate $M$, and final mass of $\mathrm{PBH}$ is

$$
M=\frac{M_{0}}{1-\eta} .
$$

For estimation of $\eta$ we use results of solution of nonstationary spherically symmetric problem obtained in paper [7. As follows from the Table in that work, the mass $M_{0}$ of formed PBH doesn't exceed 10 per cent of horizon mass $M_{h o r}$ at the time $t_{0}$ for all investigated in [7] variants. The coefficient $\eta$ is uniquely defined by relation $M_{0} / M_{h o r}$ :

$$
\begin{aligned}
& \rho_{0}=\frac{3}{32 \pi G t_{0}^{2}}, \quad M_{\text {hor }}=\frac{4 \pi}{3} \rho_{0}\left(c t_{0}\right)^{3}=\frac{1}{8} \frac{c^{3} t_{0}}{G}, \\
& \eta=\frac{81}{32} \frac{G M_{0}}{c^{3} t_{0}}=\frac{81}{256} \frac{M_{0}}{M_{\text {hor }}} .
\end{aligned}
$$

Taking into account results [7, we obtain $\eta \approx 0.03$.

As shown in paper [9], in case of accretion of the isotropic photon flow (or other ultrarelativistic particles) to rotating $\mathrm{PBH}$, an evolution of specific angular momentum depending on mass can be written approximately in form $a \propto M^{-10 / 3}$, i.e. $a=a_{0}\left(\frac{M_{0}}{M}\right)^{10 / 3}$. Under $\eta \ll 1$ we obtain approximate dependences for increase of mass and decrease of PBH angular momentum during the accretion:

$$
M \approx M_{0}(1+\eta), \quad a \approx a_{0}\left(1-\frac{10}{3} \eta\right) .
$$

Under $\eta \sim 0.03$ we obtain that PBH mass $M$ increases by $\sim 3$ per cent due to accretion, and its specific angular momentum $a$ decrease by $\sim 10$ per cent.

Thus, PBH rapidly rotating at the time of formation will keep their rapid rotation. If mass of $\mathrm{PBH}$ is not too large $\left(M \sim 10^{15} \mathrm{~g}\right)$ their evaporation becomes significant by the present time [12]. Ratio between different types of particles generated during the evaporation depends essentially on angular momentum of PBH [12, what influences on estimates of different components of the background of modern Universe, such as neutrino, gravitons, and other weakly interacting particles [13].

\section{Acknowledgement}

This work was partially supported by RFBR grant 0502-17697, RAN Program "Formation and evolution of stars and galaxies" and Grant for Leading Scientific Schools NSh-10181.2006.2.

\section{Список литературы}

[1] Ya.B. Zel'dovich and I.D. Novikov, Astronomicheskii Zhurnal 43, 758 (1966) (in russian); Ya.B. Zel'dovich and I.D. Novikov, Soviet Astronomy, 10, 602 (1967) (in english).

[2] D. Carr and S.L. Hawking, Mon. Not. R. Astron. Soc. 168, 399 (1974).

[3] Ya.B. Zel'dovich and I.D. Novikov, "The Relativistic Astrophysics Nauka, Moscow, 1967 (in russian).

[4] Ya.B. Zel'dovich and I.D. Novikov, "Gravitation Theory and Star Evolution Nauka, Moscow, 1971 (in russian).

[5] A.G. Polnarev, M.Yu. Khlopov, Uspekhi Fizicheskikh Nauk 145, 369 (1985) (in russian).

[6] M.Yu. Khlopov, R.V. Konoplich, S.G. Rubin and A.S. Sakharov, Gravitation and Cosmology 6, 153 (2000). 
[7] D.K. Nadezhin, I.D. Novikov and A.G. Polnarev, Astronomicheskii Zhurnal 55, 216 (1978) (in russian); D.K. Nadezhin, I.D. Novikov and A.G. Polnarev, Soviet Astronomy 22, 129 (1978) (in english).

[8] A.G. Doroshkevich, Astronomicheskii Zhurnal 43, 105 (1966) (in russian); A.G. Doroshkevich, Soviet Astronomy 10, 83 (1966) (in english).

[9] P.J. Young, Phys. Rev. D 14, 3281(1976).

[10] P.J. Young, ApJ 212, 227 (1977).

[11] G.S. Bisnovatyi-Kogan and O.Yu. Tsupko, Astrofizika, 50, 653 (2007) (in russian).

[12] V.P. Frolov and I.D. Novikov, "Black Hole Physics: Basic Concepts and New Developments Kluwer Academic Press, Dordrecht, 1998.

[13] G.S. Bisnovatyi-Kogan and V.N. Rudenko, Classical and Quantum Gravity 21, 3347 (2004). 